\title{
AUTOMORPHISMS OF SEMIGROUPS OF CONTINUOUS FUNCTIONS
}

\author{
G. R. WOOD \\ (Received 14 November 1978; revised 21 September 1979) \\ Communicated by T. E. Hall
}

\begin{abstract}
Certain semigroups of continuous selfmaps of the closed unit interval are shown to have the property that all their automorphisms are inner. Contrary to expectation, certain other such semigroups do have outer automorphisms.
\end{abstract}

1980 Mathematics subject classification (Amer. Math. Soc.) : primary 20 M 20; secondary 54 C 40.

\section{Introduction}

For many transformation semigroups, with the operation of function composition, it is known that all automorphisms are inner. However, general results of this type are few : proofs usually apply in an ad hoc fashion.

A recent encouraging advance is the general result of Fitzpatrick and Symons (1975). They have shown that if $X$ is an infinite set, and $\mathscr{S}$ a semigroup of total transformations which contains $\mathscr{G}$, the symmetric group on $X$, then $\mathscr{S}$ has the inner automorphism property. The proof uses the fact that the property is known for $\mathscr{G}$. Another general result has been established by Sullivan (1975) for subsemigroups of $\mathscr{P}$, the semigroup of all partial transformations of the set $X$. A subsemigroup $\mathscr{S}$ of $\mathscr{P}$ is said to cover $X$ if, for each $x$ in $X$, there exists some constant idempotent in $\mathscr{S}$ with range $x$. All automorphisms, $\varphi$, of such semigroups are shown to be of the form

$$
\varphi(f)=h f h^{-1}, \text { for all } f \text { in } \mathscr{S},
$$

where $h$ is an element of $\mathscr{G}$.

The purpose of this paper is to investigate the case where $X=[0,1]=I$, the closed unit interval, with $\mathscr{S}$ throughout a semigroup of continuous functions from $I$ to itself, containing $\mathscr{G}$, the group of all homeomorphisms of $I$. Fine and Schweigert (1955) have investigated $\mathscr{G}$ and shown that all its automorphisms are inner. 
Curiously, the analogue of Fitzpatrick and Symons' result does not hold : there exist semigroups $\mathscr{S}$ with both inner and outer automorphisms. However, certain general results can be obtained indicating that, for example, the semigroup of all one-to-one continuous functions $(\mathscr{M})$ and the semigroup of all onto continuous functions ( $\mathscr{E})$ both have the inner automorphism property.

Subsemigroups of $\mathscr{T}$ (the semigroup of all continuous selfmaps of $I$ ) have already been considered by Gluskin (1959). He establishes the inner automorphism property for $\mathscr{M}$, all strictly monotone functions in $\mathscr{T}$. It is interesting to note that the proof does incorporate the Fine and Schweigert result. Gluskin also studies the semigroups

(i) $\mathscr{D}$, all increasing functions in $\mathscr{M}$,

(ii) $\mathscr{B}_{0}$, all $f \in \mathscr{D}$ such that $f(0)=0$ and $f(1)<1$, and

(iii) $\mathscr{C}_{0}$, all $f \in \mathscr{B}_{0}$ which are the identity on some interval containing the point 0 . He proves that every automorphism $\varphi$ of $\mathscr{D}$ has the form $\varphi(f)=h f h^{-1}$, for all $f$ in $\mathscr{D}$, where $h$ is an element of $\mathscr{G}$, while for $\mathscr{B}_{0}\left(\mathscr{C}_{0}\right)$ all automorphisms have the form $\varphi(f)=h f h^{-1}$, for all $f$ in $\mathscr{B}_{0}\left(\mathscr{C}_{0}\right)$, where $h$ is an element of $\mathscr{G} \cap \mathscr{D}$.

A more general framework, with $X$ a closed, bounded subset of $\mathbf{R}^{n}$ and $\mathscr{M}$ the semigroup of all homeomorphisms from $X$ into $X$, is considered in Gluskin (1960). He investigates the automorphisms of certain subsemigroups of $\mathscr{M}$.

Even a brief survey must mention some of the results of Magill. Endomorphisms of $\mathscr{T}$ have been characterized in Magill (1977) as being of two types : either an endomorphism $\varphi$ sends every element of $\mathscr{T}$ into a single idempotent, or it is injective and there exists a unique idempotent $v$ in $\mathscr{T}$ and a unique homeomorphism $h$ from $I$ onto the range of $v$ such that $\varphi(f)=h f h^{-1} v$, for every $f$ in $\mathscr{T}$.

In Cezus, Magill and Subbiah (1975) the maximal left, right and two-sided ideals of $\mathscr{T}$ are completely described.

\section{A simplification}

To show that $\mathscr{S}, \mathscr{G} \subseteq \mathscr{S} \subseteq \mathscr{T}$, has the inner automorphism property, it suffices to show that an automorphism of $\mathscr{S}$ which is the identity on $\mathscr{G}$ is necessarily the identity automorphism. The method is due to Fitzpatrick and Symons. Since the centre of $\mathscr{G}$ is trivial, Fine and Schweigert (1955), Theorem 7, the converse is certainly true.

Let $\varphi$ be an automorphism of $\mathscr{S}$. Elements in $\mathscr{G}$ are characterized in $\mathscr{S}$ by the existence of two-sided inverses in $\mathscr{S}$, so that $\varphi$ induces an automorphism of $\mathscr{G}$. We know from Fine and Schweigert (1955) that there is an $h \in \mathscr{G}$ such that

$$
\varphi(g)=h g h^{-1} \text {, for every } g \in \mathscr{G} .
$$


Now consider the automorphism $\psi$ of $\mathscr{S}$ given by

$$
\psi(f)=h^{-1} f h, \text { for every } f \text { in } \mathscr{S} .
$$

Then $\varphi \psi$, an automorphism of $\mathscr{P}$, is the identity on $\mathscr{G}$. If it were the identity on $\mathscr{P}$,

$$
f=\varphi \psi(f)=\varphi\left(h^{-1} f h\right)=h^{-1} \varphi(f) h,
$$

or $\varphi$ has the required property.

So it suffices to show that if $\varphi$ is the identity on $\mathscr{G}$, it is the identity on $\mathscr{S}$. We show this for many semigroups $\mathscr{S}$. From now, $\varphi$ will be an automorphism of $\mathscr{S}$ which is the identity on $\mathscr{G}$.

\section{Results}

Our central results take the form of two lemma-theorem pairs (Lemmas 1 and 2, Theorems 1 and 2). In each lemma we show that automorphisms preserve a certain property of functions, $f$, in $\mathscr{S}:$ in the first it is a property of the range of $f$, while in the second it is a property of the domain of $f$. These results are used in the corresponding theorems to show that if $\mathscr{S}$ contains a particular type of function, then $\mathscr{S}$ has the inner automorphism property. The duality of the two results will be highlighted in the presentation. Finally, necessary and sufficient conditions for a semigroup containing all constant functions to have the inner automorphism property are given in Theorem 3. Examples of semigroups with outer automorphisms are dispersed throughout.

The range of $f \in \mathscr{S}$ is denoted by $\mathscr{R}(f)$, the constant map onto $x \in I$, by $c_{x}$, and the set of all constant maps by $\mathscr{K}$.

Our first lemma shows, with only two exceptions, that the range is preserved under $\varphi$.

LeMma 1. For $f \in \mathscr{S}, f \neq c_{0}$ or $c_{1}, \mathscr{R}(f)=\mathscr{R}(\varphi(f))$.

Proof. Assume that $f$ is not equal to $c_{0}$ or $c_{1}$ and is not onto, with $\mathscr{R}(f)=[a, b]$ (where possibly $a=b$ ). We shall show that $\mathscr{R}(\varphi(f)) \subseteq \mathscr{R}(f)$. To do this, choose $g \in \mathscr{G}$ such that $g \mid[a, b]$ ( $g$ restricted to $[a, b])$ is the identity, $g$ fixes $\{0,1\}$ and moves all other elements of $[0,1]$. Then $g f=f$, so that $g \varphi(f)=\varphi(f)$ and $g \mid \mathscr{R}(\varphi(f))$ is the identity. If $\mathscr{R}(\varphi(f))=\{0\}$, then $\varphi(f)=c_{0}$ and so (Sullivan (1975), Lemmas 1 and 2) $f$ is constant, $c_{a}$ say $(a \neq 0,1)$. Choose $h \in \mathscr{G}$ where $(h(0)=0) h(a)=b \neq a$ and is otherwise arbitrary. Then $c_{0}=h c_{0}=\varphi\left(h c_{a}\right)=\varphi\left(c_{b}\right)$ provides a contradiction. Likewise $\mathscr{R}(\varphi(f)) \neq\{1\}$ and so from the definition of $g$, we obtain $\mathscr{R}(\varphi(f)) \subseteq \mathscr{R}(f)$. Since therefore $\varphi(f)$ is not onto and not equal to $c_{0}$ or $c_{1}$, by using $\varphi^{-1}$ we also have $\mathscr{R}(f) \subseteq \mathscr{R}(\varphi(f))$. Finally, it is now clear that if $f$ is not onto, neither is $\varphi(f)$, and so (using $\varphi^{-1}$ ), if $f$ is onto, then $\varphi(f)$ is also. 
The lemma does not rule out the possibility that $\varphi\left(c_{0}\right)=c_{1}$. This suggests the direction in which to look for a semigroup $\mathscr{S}$ with an outer automorphism.

Let $\mathscr{S}=\mathscr{G} \cup \mathscr{K}, h(0)=1, h(1)=0$, and $h(x)=x$ for $x$ in the open interval $(0,1)=I^{0}$. Then $\varphi$ given by $\varphi(f)=h f h^{-1}$ for every $f$ in $\mathscr{G} \cup \mathscr{K}$ is an automorphism of $\mathscr{G} \cup \mathscr{K}$ which is certainly not inner : all constant maps lie in $\mathscr{G} \cup \mathscr{K}$, so that $\varphi$ cannot be given as conjugation by any other bijection of $I$.

A point $x$ in the domain of $f \in \mathscr{S}$ is either a point of constancy or a point of variability. That is, either there exists a neighbourhood of $x$ on which $f$ is constant, or $f\left(x^{\prime}\right) \neq f(x)$ for some $x^{\prime}$ in each neighbourhood of $x$. Note that non-constant functions in $\mathscr{T}$ are determined by their values at points of variability in $I^{0}$.

Our first theorem assumes the existence, in $\mathscr{S}$, of a non-constant $s$ such that $\mathscr{R}(s) \subseteq I^{0}$. Note that if $0<a<b<1$ it is then easy to select a $g$ in $\mathscr{G}$ for which $\mathscr{R}(g s)=[a, b]$.

We now present the first result showing that a broad class of subsemigroups of $\mathscr{T}$ do have the inner automorphism property.

THEOREM 1. Let $\mathscr{S} \cap \mathscr{K}=\emptyset$. If there exists an $s$ in $\mathscr{S}$ with $\mathscr{R}(s) \subseteq I^{0}$, then every automorphism of $\mathscr{S}$ is inner.

Proof. Take $f$ in $\mathscr{S}$ and $x$ a point of variability of $f$ in $I^{0}$. Suppose that $f(x)=y \neq y^{\prime}=\varphi(f)(x)$. For the moment we assume $y \in I^{0}$ (in which case $y^{\prime}$ possibly equals 0 or 1). Choose open sets in $I, U$ and $U^{\prime}$, such that $y \in U, y^{\prime} \in U^{\prime}$ and $U \cap U^{\prime}=\emptyset$. Since $f$ and $\varphi(f)$ are continuous we can find open sets $V$ and $V^{\prime}$ about $x$, such that $f(V) \subseteq U$ and $\varphi(f)\left(V^{\prime}\right) \subseteq U^{\prime}$.

Now choose $p$ in $\mathscr{S}$ such that $V \cap V^{\prime} \supseteq \mathscr{R}(p)=\mathscr{R}(\varphi(p))$, possible by a fact noted above, and choose $g$ in $\mathscr{G}$ to be the identity on $U^{\prime}$, yet nowhere the identity on $U$ (here we need $y \neq 0$ or 1$)$.

Clearly $\varphi(f) \varphi(p)=g \varphi(f) \varphi(p)$, yet $f p \neq g f p$, a contradiction. Hence $y=y^{\prime}$. If $y^{\prime}$ equals 0 or 1 , the previous argument leads to a contradiction of the assumption $y \in I^{0}$, and so in this case we must also have $y^{\prime} \in I^{0}$. If $y=0$ or 1 , we can find a sequence $\left(x_{n}\right)$ in $I^{0}$, each $f\left(x_{n}\right)$ neither 0 nor 1 , converging to $x, \operatorname{so} \varphi(f)\left(x_{n}\right)=f\left(x_{n}\right)$ for each $n$. Thus

$$
\varphi(f)(x)=\lim _{x_{n} \rightarrow x} \varphi(f)\left(x_{n}\right)=\lim _{x_{n} \rightarrow x} f\left(x_{n}\right)=f(x),
$$

so $\varphi(f)(x)=f(x)$ at each interior point of variability, $x$. From our earlier remarks we know that this implies $\varphi(f)=f$, as required.

Given that $\mathscr{R}(p)=\mathscr{R}(\varphi(p))$, the diagrams in Figure 1 are an aid to visualizing, in a particular case, that $f g \neq g f p$, while $\varphi(f) \varphi(p)=g \varphi(f) \varphi(p)$. 

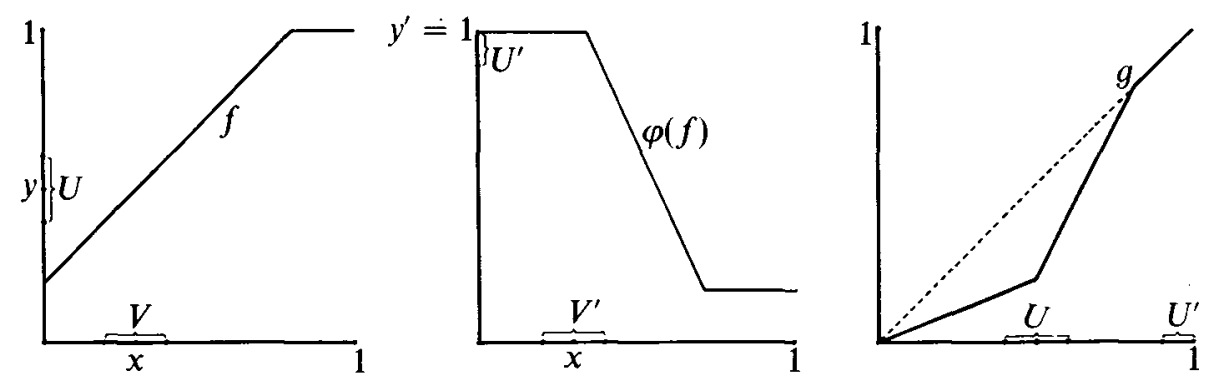

Figure 1.

Comment. Theorem 1 includes the case $\mathscr{S}=\mathscr{M}$, previously handled by Gluskin (1959).

\section{EXAMPLE. Let}

$$
\begin{aligned}
& \mathscr{R}^{\prime}=\left\{f \in \mathscr{T}: \mathscr{R}(f) \subseteq I^{0}, f(0)=f(1) \text { and each } x \text { in } I\right. \\
&\text { is a point of variability of } f\} .
\end{aligned}
$$

Theorem 1 shows that every automorphism of $\mathscr{R}^{\prime} \cup \mathscr{G}$ is inner. However, the automorphism $\varphi$ given by $\varphi(f)=h f h^{-1}$, for every $f$ in $\mathscr{R}^{\prime} \cup \mathscr{G} \cup \mathscr{K}$, with $h$ as in the previous example, is outer.

A closed interval $F$ in $I$ is termed a line of constancy of $f$ in $\mathscr{S}$ if $f$ is constant on $F$. Our second lemma shows that lines of constancy are preserved by an automorphism.

LEMMA 2. Let $f \in \mathscr{S}$. Then $F$ is a line of constancy for $f$ if and only if $F$ is a line of constancy for $\varphi(f)$.

Proof. Take distinct $x$ and $y$ in the interior of $F, F^{0}$. Let $g$ in $\mathscr{G}$ be such that $g$ moves every element of $F^{0}, g(y)=x$ and $g$ on $I \backslash F^{0}$ is the identity.

Then $f g=f$, so $\varphi(f) g(y)=\varphi(f) y$ or $\varphi(f) x=\varphi(f) y$. Since $\varphi(f)$ is constant on $F^{0}$, it is constant on $F$. Conversely, if $\varphi(f)$ is constant on $F, \varphi^{-1}(\varphi(f))=f$ is constant on $F$.

We now present the second general result.

THEOREM 2. Let $\mathscr{S} \cap \mathscr{K}=\varnothing$. If there exists a function $k$ in $\mathscr{S}$ with a line of constancy, every automorphism of $\mathscr{S}$ is inner.

Proof. As before, we take $f$ in $\mathscr{S}$ and $x$ a point of variability of $f$ in $I^{0}$. Suppose that $f(x)=y \neq y^{\prime}=\varphi(f)(x)$ and $y \in I^{0}$. It is always possible to find a $g$ in $\mathscr{G}$ such that 
$k g\left(=k^{\prime}\right)$ is constant in a neighbourhood of $y$ and not so at $y^{\prime}$ (here we need $y \in I^{0}$ ) or vice-versa. Without loss of generality we assume the former. The diagrams in Figure 2 indicate in a particular instance why there exists a $k^{\prime}$ in $\mathscr{S}$ of the desired form.
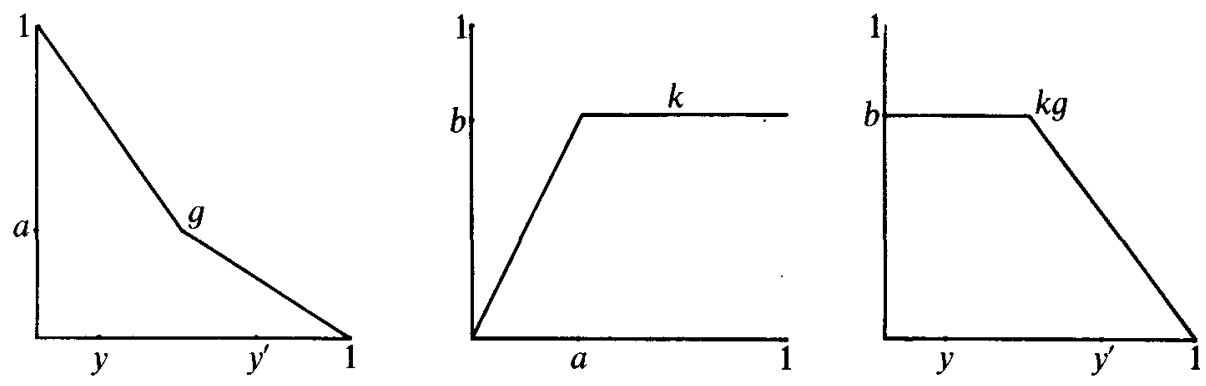

FIGURE 2.

Note that no increasing $g$ will suffice in this example and that in general (unlike the proof of Theorem 1) it does not matter whether $y^{\prime}$ equals 0 or 1 .

Observe now that $k^{\prime} f$ will be constant on some closed interval about $x, F^{\prime}$ say, while $\varphi\left(k^{\prime}\right) \varphi(f)$ will have a point of variability at $x$. This at once contradicts lemma 2. However, in order to stress the dual nature of Theorems 1 and 2, we complete the proof in the following way. If $g \in \mathscr{G}$ is other than the identity on $F^{\prime}$ but $g \mid X \backslash F^{\prime}$ is the identity, then we have $k^{\prime} f g=k^{\prime} f$, while $\varphi\left(k^{\prime}\right) \varphi(f) g \neq \varphi\left(k^{\prime}\right) \varphi(f)$, a contradiction. Hence $\varphi(f) x=f(x)$ at points of variability and so $\varphi(f)=f$. //

COMMENT. Theorem 2 includes the case $\mathscr{S}=\mathscr{E}$, the semigroup of all continuous onto mappings.

EXAMPLE. Let

$$
\begin{aligned}
& \mathscr{U}=\left\{f \in \mathscr{T} \backslash \mathscr{G}: f\left(I^{0}\right) \subseteq I^{0} \text { and either } f(0)=0, f(1)=1\right. \text { or } \\
& \qquad f(0)=1, f(1)=0\} .
\end{aligned}
$$

Theorem 2 shows that every automorphism of $\mathscr{G} \cup \mathscr{U}$ is inner while a similar construction to that earlier shows that $\mathscr{G} \cup \mathscr{U} \cup \mathscr{K}$ has an outer automorphism.

Semigroups dealt with in Theorems 1 and 2 have excluded the constant maps. We turn finally to the case of semigroups $\mathscr{S}$ which contain $\mathscr{G} \cup \mathscr{K}$. Consider the following subsets of $\mathscr{T}$ :

$$
\begin{aligned}
\mathscr{P} & =\{f \in \mathscr{T} \backslash \mathscr{K}: f(0)=f(1) \text { and equals } 0 \text { or } 1\}, \\
\mathscr{Q} & =\left\{f \in \mathscr{T} \backslash \mathscr{K}: f(0)=f(1) \in I^{0} \text { and } f(x) \text { equals } 0 \text { or } 1 \text { for some } x \text { in } I^{0}\right\}, \\
\mathscr{R} & =\left\{f \in \mathscr{T} \backslash \mathscr{K}: f(0)=f(1) \text { and } \mathscr{R}(f) \subseteq I^{0}\right\},
\end{aligned}
$$


$\mathscr{V}=\{f \in \mathscr{T}: f(0)=0, f(1)=1$ or $f(0)=1, f(1)=0$, and $f(x)$ equals 0 or 1 for some $x$ in $\left.I^{0}\right\}$

$\mathscr{W}=\left\{f \in \mathscr{T}: f(0) \neq f(1)\right.$ with $f(0)$ or $f(1)$ in $\left.I^{0}\right\}$.

Now $\mathscr{G}, \mathscr{K}, \mathscr{P}, \mathscr{Q}, \mathscr{R}, \mathscr{U}, \mathscr{V}$ and $\mathscr{W}$ are disjoint sets whose union is $\mathscr{T}$. This partitioning of $\mathscr{T}$ enables us to prove the final theorem.

THEOREM 3. Let $\mathscr{G} \cup \mathscr{K} \subseteq \mathscr{S}$. Then every automorphism of $\mathscr{S}$ is inner if and only if $\mathscr{S}$ contains an element of $\mathscr{P} \cup \mathscr{Q} \cup \mathscr{V} \cup \mathscr{W}$.

PRoof. It follows from Schreier (1937) that an automorphism $\varphi$ of $\mathscr{S}$ has the form

$$
\varphi(f)=h f h^{-1}, \quad \text { for all } f \text { in } \mathscr{S},
$$

where the bijection $h$ of $I$ is determined uniquely by $\varphi\left(c_{x}\right)=c_{h(x)}$, for each $x$ in $I$. From Lemma 1 we know that if $\varphi$ is the identity on $\mathscr{G}, \varphi\left(c_{x}\right)=c_{x}$ and so $h(x)=x$, for each $x$ in $I^{0}$.

Now suppose $\mathscr{S}$ contains an element $f$ in $\mathscr{P} \cup \mathscr{Q} \cup \mathscr{V} \cup \mathscr{W}$. If $h$ were to reverse 0 and $1, h f h^{-1}$ is readily shown to be discontinuous, hence $h$ can only be the identity, and so every automorphism is inner.

On the other hand, if $\mathscr{G} \cup \mathscr{K} \subseteq \mathscr{S} \subseteq \mathscr{G} \cup \mathscr{K} \cup \mathscr{R} \cup \mathscr{U}$, and $h$ reverses 0 and 1 but is the identity on $I^{0}$, then

$$
h f h^{-1}= \begin{cases}f & \text { if } f \in \mathscr{G} \cup \mathscr{R} \cup \mathscr{U}, \\ c_{0} & \text { if } f=c_{1}, \\ c_{1} & \text { if } f=c_{0} .\end{cases}
$$

Thus $\varphi$, given as conjugation by $h$, is an automorphism of $\mathscr{S}$ which is not inner. //

COROLLARY. $\mathscr{T}$ has the inner automorphism property, a special case of Magill (1964), (3.5).

ObSERVATIONs. 1. Other topological spaces. In the case of $I^{0}$, Theorem 1 (with the closure of $\mathscr{R}(s) \subseteq I^{0}$ replacing $\mathscr{R}(\mathrm{s}) \subseteq I^{0}$ in the statement) and Theorem 2 hold. This rests on the fact that $\mathscr{G}\left(I^{0}\right)$, the group of homeomorphisms of $I^{0}$, is isomorphic to $\mathscr{G}(I)$, and hence has the inner automorphism property. It follows from the first paragraph in the proof of Theorem 3 that if $\mathscr{S}\left(I^{0}\right)$ contains all the constant maps, all automorphisms will be inner. Since $I^{0}$ is homeomorphic to $\mathbf{R}$, corresponding results also hold for $\mathbf{R}$. In particular, $\mathscr{M}(X)$ and $\mathscr{E}(X)$ both have the inner automorphism property, for $X=I, I^{0}$ and $\mathbf{R}$. Whether the Fitzpatrick and Symons result holds for $I^{0}$ remains an open question. 
2. $\mathscr{M}(X)$ and $\mathscr{E}(X)$. It is interesting to note that while $\mathscr{M}(I) \cong \mathscr{M}\left(I^{0}\right) \cong \mathscr{M}(\mathbf{R})$ and $\mathscr{E}\left(I^{0}\right) \quad \mathscr{E}(\mathbf{R}), \mathscr{E}(I) \nsubseteq \mathscr{E}\left(I^{0}\right)$. That is, $\mathscr{E}(I)$ and $\mathscr{E}\left(I^{0}\right)$ are non-isomorphic semigroups.

The proof is by contradiction, as follows. For brevity, let $\mathscr{E}(I)=\mathscr{E}, \mathscr{E}\left(I^{0}\right)=\mathscr{E}^{\circ}$ and similarly for $\mathscr{G}$ and $\mathscr{K}$. Suppose $\varphi$ is an isomorphism from $\mathscr{E}$ onto $\mathscr{E}^{0}$. Then $\varphi(\mathscr{G})=\mathscr{G}^{0} \cong \mathscr{G}$, so for some $h \in \mathscr{G}$ we may express $\varphi$ as

$$
\varphi(g)=h g h^{-1} \mid I^{0}, \quad \text { for all } g \in \mathscr{G} \text {. }
$$

A modification of the method used in Section 2 allows us to assume that our isomorphism takes $g \in \mathscr{G}$ to its restriction in $\mathscr{G}^{0}$. Then, as in Lemma 2, we may show that if $f \in \mathscr{E}$ has a line of constancy $F \subseteq I^{0}$, so too will $\varphi(f)$ in $\mathscr{E}^{0}$.

If we define

$$
\mathscr{A}=\left\{f \in \mathscr{E}: f \text { is constant on some } F \subseteq I^{0}\right\}
$$

and

$$
\mathscr{A}^{0}=\left\{f \in \mathscr{E}^{0}: f \text { is constant on some } F \subseteq I^{0}\right\},
$$

then we have $\varphi(\mathscr{A})=\mathscr{A}^{0}$.

Now for a fixed closed interval $F^{\prime} \subseteq I^{0}, \mathscr{E}$ has the following property : there exists an $f$ in $\mathscr{E}$ (any $f \in \mathscr{E}$ such that $f\left(F^{\prime}\right)=I$ will suffice) such that for all $k$ in $\mathscr{A}, k f$ is not constant on $F^{\prime}$. On the other hand, $\mathscr{E}^{\circ}$ has the property : for all $f$ in $\mathscr{E}^{\circ}$, there exists a $k \in \mathscr{A}^{0}$ such that $k f$ is constant on $F^{\prime}$. This is a contradiction.

3. The differentiable case. Let $\mathscr{G}$ be all diffeomorphisms of $I$. As before, we can construct an outer automorphism of $\mathscr{G} \cup \mathscr{K}$. With suitable smoothing of the functions used in the proofs for the continuous case, we could show that Theorems 1, 2 and 3 hold, should $\mathscr{G}$ have the inner automorphism property. To the best of the author's knowledge this last result is yet unproved.

\section{Acknowledgements}

The author is most grateful to Dr. R. P. Sullivan for a number of encouraging conversations, as well as for pointing out the works of Gluskin. During the later stages of the work the comments of Dr. W. Barit concerning the duality of Theorems 1 and 2 were also very much appreciated. Thanks are also due to the referee, for a most thorough appraisal of the paper.

\section{References}

F. A. Cezus, K. D. Magill, Jr. and S. Subbiah (1975), 'Maximal ideals of semigroups of endomorphisms', Bull. Austral. Math. Soc. 12, 211-225. 
N. J. Fine and G. E. Schweigert (1955), 'On the group of homeomorphisms of an arc', Ann. of Math. 62, 237-253.

S. P. Fitzpatrick and J. S. V. Symons (1975), 'Automorphisms of transformation semigroups', Proc. Edinburgh Math. Soc. 19, 327-329.

L. M. Gluskin (1959), 'The semigroup of homeomorphic mappings of an interval', Mat. Sb. (N.S.) 49 (91), 13-18; translated in Amer. Math. Soc. Transl. 30, (1963), 273-290.

L. M. Gluskin (1960), 'Automorphisms of semigroups of topological mappings', Izv. Vyss. Ucebn. Zaved. Matematika 6 (19), 62-73; translated in Amer. Math. Soc. Transl. 36 (1964), 383-395.

K. D. Magill, Jr. (1964). 'Semigroups of continuous functions', Amer. Math. Monthly 71, 984-988.

K. D. Magill, Jr. (1977), 'Homomorphisms from $\mathscr{S}(X)$ into $\mathscr{P}(Y)$ ', Can. J. Math. 29 615-625.

J. Schreier (1937), 'Uber Abbildungen einer abstrakten Menge auf ihre Teilmengen', Fund. Math. 28, 261-264.

R. P. Sullivan (1975), 'Automorphisms of transformation semigroups', J. Austral. Math. Soc. Ser. A 20, $77-84$.

Department of Mathematics

University of Canterbury

Christchurch

New Zealand 